\title{
Cochrane
}

Cochrane Database of Systematic Reviews

\section{Education and training for preventing and minimising workplace aggression directed toward healthcare workers (Protocol)}

Hills DJ, Ross HM, Pich J, Hill AT, Dalsbø TK, Riahi S, Guay S, Martínez-Jarreta B

Hills DJ, Ross HM, Pich J, Hill AT, Dalsbø TK, Riahi S, Guay S, Martínez-Jarreta B.

Education and training for preventing and minimising workplace aggression directed toward healthcare workers.

Cochrane Database of Systematic Reviews 2015, Issue 9. Art. No.: CD011860.

DOI: 10.1002/14651858.CD011860.

www.cochranelibrary.com

Education and training for preventing and minimising workplace aggression directed toward healthcare workers (Protocol) Copyright $\odot 2015$ The Cochrane Collaboration. Published by John Wiley \& Sons, Ltd. 
TABLE OF CONTENTS

HEADER . . . . . . . . . . . . . . . . . . . . . . . . . . . . . . . . . . . . . . . . 1

ABSTRACT . . . . . . . . . . . . . . . . . . . . . . . . . . . . . . . . . . . . . . . 1

BACKGROUND . . . . . . . . . . . . . . . . . . . . . . . . . . . . . . . . . . . .

OBJECTIVES . . . . . . . . . . . . . . . . . . . . . . . . . . . . . . . . . . . . . . . . . .

METHODS . . . . . . . . . . . . . . . . . . . . . . . . . . . . . . . . . . . . . . 44

ACKNOWLEDGEMENTS . . . . . . . . . . . . . . . . . . . . . . . . . . . . . . . . . . . . . . . . . .

REFERENCES . . . . . . . . . . . . . . . . . . . . . . . . . . . . . . . . . . . . . . . . .

APPENDICES . . . . . . . . . . . . . . . . . . . . . . . . . . . . . . . . . . . . . 12

CONTRIBUTIONS OF AUTHORS . . . . . . . . . . . . . . . . . . . . . . . . . . . . . . . . . . . . . . 17

DECLARATIONS OF INTEREST . . . . . . . . . . . . . . . . . . . . . . . . . . . . . . . . 17

Education and training for preventing and minimising workplace aggression directed toward healthcare workers (Protocol)

Copyright $\odot 2015$ The Cochrane Collaboration. Published by John Wiley \& Sons, Ltd. 


\title{
[Intervention Protocol]
}

\section{Education and training for preventing and minimising workplace aggression directed toward healthcare workers}

\author{
Danny J Hills ${ }^{1}$, Heather M Ross ${ }^{2}$, Jacqueline Pich ${ }^{3}$, April T Hill ${ }^{2}$, Therese K Dalsbø ${ }^{4}$, Sanaz Riahi ${ }^{5}$, Stéphane Guay ${ }^{6}$, Begoña Martínez- \\ Jarreta $^{7}$ \\ ${ }^{1}$ Disciplines of Nursing and Midwifery, Faculty of Health, University of Canberra, Canberra, Australia. ${ }^{2}$ College of Nursing and Health \\ Innovation, Arizona State University, Phoenix, AZ, USA. ${ }^{3}$ School of Nursing and Midwifery, University of Newcastle, Callaghan, \\ Australia. ${ }^{4}$ Norwegian Knowledge Centre for the Health Services, Oslo, Norway. ${ }^{5}$ Professional Practice \& Clinical Information, Ontario \\ Shores Centre for Mental Health Sciences, Whitby, Canada. ${ }^{6}$ School of Criminology, University of Montreal, Montreal, Canada. \\ ${ }^{7}$ Department of Forensic Medicine and Toxicology. Faculty of Medicine, University of Zaragoza, Zaragoza, Spain
}

Contact address: Danny J Hills, Disciplines of Nursing and Midwifery, Faculty of Health, University of Canberra, Canberra, ACT, 2601, Australia.danny.hills@canberra.edu.au.

Editorial group: Cochrane Work Group.

Publication status and date: New, published in Issue 9, 2015.

Citation: Hills DJ, Ross HM, Pich J, Hill AT, Dalsbø TK, Riahi S, Guay S, Martínez-Jarreta B. Education and training for preventing and minimising workplace aggression directed toward healthcare workers. Cochrane Database of Systematic Reviews 2015, Issue 9. Art. No.: CD011860. DOI: 10.1002/14651858.CD011860.

Copyright (C) 2015 The Cochrane Collaboration. Published by John Wiley \& Sons, Ltd.

\section{A B S T R A C T}

This is the protocol for a review and there is no abstract. The objectives are as follows:

To assess the effectiveness of education and training interventions that aim to prevent and minimise workplace aggression directed toward healthcare workers by patients and patient advocates.

\section{B A C K G R O U N D}

Aggression is a common characteristic of human behaviour and a significant feature of contemporary society. Fundamentally, human aggression is social behaviour. It requires a social context in which some form of verbal and non-verbal interaction may take place between at least two people (Cherek 2003). Most people would have both experienced and engaged in aggression. Aggression is not to be confused, however, with what might more accurately be termed confidence, enthusiasm or even assertiveness (Bushman 2001). The function of aggression in humans in contemporary societies can be considered in terms of the extent to which it is adaptive or maladaptive. From an evolutionary perspective, aggression can be seen to relate primarily to attempts to pro- tect the self or significant others in response to a localised threat or danger, and in response to the competition for resources (Archer 1988). It is also clear, however, that aggression is often harmful and maladaptive in contemporary human society, and is more commonly considered non-normative, particularly in relation to more extreme forms of aggression such as physical and sexual assault.

\section{Description of the condition}

On an individual level, aggression may extend from relatively mild, verbal expressions of dissatisfaction, frustration or hostility through to extreme acts of violence. Aggression may be used to coerce, intimidate or exert power over another. It may be di- 
rected toward inanimate objects or toward other living beings. Aggression may be directed toward peers, authority figures, subordinates or complete strangers. It may be directed toward people with wealth and prestige, or toward people less fortunate, less capable of mounting a defence or deemed sufficiently different, because of socioeconomic status, disability, gender, sexual orientation, race, ethnicity or religion, for example. It may also extend to group forms, such as mobbing.

\section{Aggression in the workplace}

Aggression also commonly occurs within the interactional context of work. It is a surprisingly prevalent phenomenon across the globe, with data from the US, Australia, Japan, Saudi Arabia and Malaysia indicating that large numbers of working people, in a range of occupations, experience aggression from multiple sources at work (di Martino 2005). Aggression may be employed by people external to the workplace (customers/clients and other members of the public) or internal to the workplace (supervisors and other co-workers) to express more immediate distress, frustration or hostility, or more deliberately and systematically coerce, intimidate, discriminate or exert power. Overall, however, aggression from external sources is more prevalent than aggression from coworkers (Cookson 2012; Harrell 2011; LeBlanc 2002; LeBlanc 2006; Packham 2011). There is a large body of evidence relating to exposure to workplace aggression from a range of sources and subsequent adverse consequences for individuals and organisations. This includes relatively short-lived feelings of distress, fear and shame, through to longer-term impacts on physical and mental health for individuals (Briggs 2003; Brown 2011; Flannery 2001; Hershcovis 2010; Hills 2014; Hinduja 2007; Hogh 2005a; Hogh 2005b; LeBlanc 2002; Mayhew 2007; Niedhammer 2009; Wieclaw 2006), and impacts on their home lives (Lewis 2005). Workplace aggression exposure is also associated with adverse work-related outcomes, including in relation to job satisfaction, organisational commitment and workforce participation intentions (Dupré 2014; Heponiemi 2014; Hills 2014; Lanctôt 2014; Lapierre 2005; LeBlanc 2002). In the healthcare sector, there is some evidence of health worker exposure to workplace aggression also impacting on the quality and safety of health care (Arnetz 2001; Laschinger 2014; Paice 2009; Rosenstein 2008).

\section{Aggression in health care}

The process of delivering health care comprises often complex interactions with patients, their advocates, co-workers and a range of other people peripherally associated or completely unconnected with service delivery (e.g. intruders). It is often stressful work, typically involving working with people who are experiencing distressing conditions or circumstances and sub-optimal cognition, affect or arousal. Consequently, it would be expected that aggression is likely to be an unwelcome feature of healthcare work. Indeed, people working in health care are at high risk of experiencing workplace aggression, second only to people working in protection and security services (Cookson 2012; di Martino 2002; Estrada 2010; Parent-Thirion 2007; Packham 2011). Furthermore, health workers can be exposed to other occupational conditions associated with a higher risk for experiencing workplace aggression, including working alone or in small numbers, working at night and working in acute care community-based settings (Bulatao 1996; Chappell 2006; Mayhew 2000; Wiskow 2003).

Workplace aggression in health care has become a widely researched phenomenon. This is important, since there is a good deal of evidence that poor reporting practices are the norm rather than the exception in healthcare settings (Farrell 2006; Judy 2009; Mayhew 2001; Parker 2010). Organisational data are dependent on voluntary reporting by staff, yet there is a significant problem with under-reporting of incidents due to a lack of clarity about what is a reportable incident, or organisational culture or inadequate support to staff reporting incidents of workplace aggression (Atawneh 2003; Gates 2011; Gerberich 2004; Kvas 2014). Aggression may be viewed by staff and employers as just being part of the job, further contributing to under-reporting (Child 2010; Ventura-Madangeng 2009). Consequently, survey research may be the most reliable method of estimating the extent of workplace aggression in healthcare settings, despite the likely limitations of recall bias and response bias.

\section{Prevalence of aggression in health care}

A major feature of workplace aggression in healthcare research published since 2000 is that the majority of studies have focused on nurses, with a smaller body of research focusing on medical practitioners or mixed populations of health workers, typically in which nurses are the majority of respondents. Most of this research has been exploratory and descriptive in nature, mostly estimating six-month, 12-month or career prevalence, using crosssectional, retrospective, self report survey designs with customised instruments unique to individual studies (Hahn 2008; Hills 2013; Taylor 2010). Such study-specific variations render efforts to establish broadly based prevalence rates extraordinarily challenging. Furthermore, the rates of different forms and sources of aggression vary considerably between nations (Camerino 2008; di Martino 2002; Spector 2014).

A further complication associated with establishing prevalence rates relates to how workplace aggression is conceptualised and defined in different studies, if explicated at all. Alternative terms include 'occupational aggression', 'occupational violence' and 'counterproductive work behaviour'. The terms 'aggression' and 'violence' are often interchanged. Of most concern is the highly problematic use of the term 'violence' to include less extreme and nonphysical forms of aggression, even though verbal or written expressions of aggression may include highly disturbing threats of violence. Additionally, it has been argued that it is important to distinguish 'resistance to care' behaviour from aggressive behaviour. 
While appearing similar, the behavioural intentions and the therapeutic responses required are clinically significantly different, with the primarily defensive 'resistance to care' being frequently exhibited by people with some form of cognitive impairment (Kable 2012). This differentiation appears not to be explicitly considered in much of the health profession workplace aggression literature. Despite the challenges of defining and establishing the extent of workplace aggression in health care, patients have been identified as the most common source of aggression, with $10 \%$ to $95 \%$ of respondents having reported experiencing verbal or physical forms of aggression from patients, with aggression from patients' advocates reported by $20 \%$ to $50 \%$ of respondents in the previous six, 12 or 24 months (Arnetz 2001; Campbell 2011; Carluccio 2010; Farrell 2006; Frank 1998; Gascón 2009; Gerberich 2004; Guay 2014; Hahn 2010; Hegney 2006; Hills 2012; Hills 2013; Hodgson 2004; Martínez-Jarreta 2007; O’Brien-Pallas 2009; Roche 2010; Spector 2014; Viitasara 2003). Where aggression from supervisors and other co-workers has been investigated, it was usually the third most common source, experienced by $3 \%$ to $40 \%$ of survey respondents (Arnetz 2001; Camerino 2008; Campbell 2011; Farrell 2006; Farrell 2010; Hegney 2006; Hills 2012; Hills 2013; Hodgson 2004; O’Brien-Pallas 2009; Roche 2010).

\section{Prevention and minimisation of workplace aggression in health care}

As a consequence of the existing evidence on the prevalence of workplace aggression and the wide range of consequences affecting individuals and organisations, there is broad agreement that a diversity of integrated approaches is required to effectively prevent and minimise aggression and its impact within organisations (ILO 2002; ILO 2003; Mayhew 2000; Mayhew 2004; McCarthy 2004; OSHA 2004; Viitasara 2002). Education and training in the prevention and minimisation of workplace aggression is a key component of any workplace aggression prevention programme but can only be considered one of a necessary range of approaches required to address this work health and safety concern. Education and training interventions are unlikely to resolve organisational systems, environmental or cultural challenges and, in any case, they need to be developed on the basis of clearly identified needs (Anderson 2010).

\section{Description of the intervention}

Education and training for the prevention and minimisation of workplace aggression may comprise any of a broad range of techniques to enhance knowledge and understanding of organisational policies and procedures, legal responsibilities, and risk assessment and control strategies. In addition, specific interpersonal skills and behaviour management techniques may be tailored to the specific work roles of personnel in the context of their workplaces
(Chappell 2006; Farrell 2005; ILO 2002; ILO 2003; Mayhew 2000; Mayhew 2001; OSHA 2004).

We will define education as the process of imparting knowledge and understanding of organisational policies and procedures, legal responsibilities, and risk assessment and control strategies, including in relation to specific techniques that may be employed in one's work to prevent and minimise the likelihood and consequences of exposure to workplace aggression. We will define training as the process of education about, and rehearsal and simulated or invivo practice of, cognitive and behavioural skills that may be implemented in one's work to prevent and minimise the likelihood and consequences of exposure to workplace aggression.

\section{How the intervention might work}

As highlighted above, education and training interventions, in isolation, are unlikely to resolve systems, environmental or cultural challenges that may impact on the likelihood and consequences of incidents workplace aggression in health service organisations. Nonetheless, by improving the knowledge, attitudes and skills of individual and groups of healthcare workers relating to the prevention and minimisation of workplace aggression directed toward them by patients and their advocates, it would be expected that the overall number of episodes of aggression, including those resulting in psychological or physical harm or injury, would be reduced. It would also be expected that the number of adverse personal and organisational outcomes attributable to incidents of workplace aggression (e.g. leave days taken, alterations to workforce participation including changing work patterns or attrition, litigation and rehabilitation costs) would be reduced.

\section{Why it is important to do this review}

The capacity to deliver purposeful, safe and effective responses to potential and escalating aggression seems essential for people engaged in any form of human service delivery, including health care, where human interactions are prominent and particularly in circumstances where the risk of aggression is more prevalent. Unfortunately, there has been a poor history of evaluation of education and training programmes in aggression minimisation and prevention (Beech 2006), Furthermore, the available evidence on the impact and outcomes of workplace aggression minimisation training programmes in diverse settings typically shows indeterminate or poor results (Bowers 2006; Gerdtz 2013; Hahn 2013; Heckemann 2015; Hills 2008; Hodgson 2004; Kansagra 2008; Laker 2010; Livingston 2010; Nachreiner 2005; Needham 2005; Price 2015). Nonetheless, clinicians and support personnel recognise its value (Arimatsu 2008; Ceramidas 2010; HEPRU 2003; HEPRU 2008; Judy 2009). Importantly, the relative absence of evidence for the effectiveness of education and training is no reason to assume that it is ineffective (Richter 2006). Indeed, in the absence of an evi- 
dence base, beneficial and possibly life-saving training may be neither sought nor provided (NICE 2006), highlighting the ongoing need for more rigorous evaluation of education and training programmes for preventing and minimising workplace aggression directed toward health workers.

While the reasons for the lack of evidence for protection afforded by education and training are unclear, it may relate, at least to some degree, to the necessary plasticity in the application of these techniques, which often need to be tailored to specific situations as they arise. Education and training in aggression minimisation and prevention is not necessarily amenable to the level of standardisation required for intervention studies. Despite these ongoing concerns, education and training is likely to remain an important component of any structured workplace aggression prevention and minimisation programme. Precisely what constitutes the key components of effective education and training in workplace aggression prevention and minimisation, however, is unclear. In this systematic review, we will examine the research evidence for the effects of all types of education and training interventions used by employers in the healthcare sector to build the knowledge or skills, or both, for participants working as individuals or groups as one means of reducing the incidence and adverse outcomes of aggression directed toward healthcare workers by patients or their advocates. This review will exclude organisational interventions, the application of physical devices or the introduction of environmental design or redesign features (including physical structures), which will be addressed in separate reviews.

\section{O B JECT IVES}

To assess the effectiveness of education and training interventions that aim to prevent and minimise workplace aggression directed toward healthcare workers by patients and patient advocates.

\section{METHODS}

\section{Criteria for considering studies for this review}

\section{Types of studies}

We will include randomised controlled trials (RCTs). In consideration of the complexity of conducting RCTs in work organisations, we will also include cluster RCTs and controlled before-and-after studies (CBAs). We will include studies where the control condition is no education and training programme for the prevention and minimisation of workplace aggression, comparison with an existing education and training programme or comparison with current practice.

\section{Types of participants}

We will include any healthcare workers of any age, gender or profession who interact with patients or their advocates, or both, in any public or private healthcare facility. This will include at least:

- physicians and physician assistants;

- dentists;

- nurses and midwives;

- allied health professionals (e.g. physiotherapists, occupational therapists, speech pathologists, medical imaging, oral hygienists, podiatrists, dietitians, opticians);

- healthcare support personnel (e.g. reception staff, healthcare aides or assistants, healthcare security personnel).

\section{Types of interventions}

We will include any educational or training intervention undertaken with healthcare workers to improve their knowledge, attitudes and skills in preventing and minimising verbal or physical aggression directed toward them and their peers in their workplace from patients or their advocates. This may include interventions designed to enhance knowledge and understanding of legal responsibilities, organisational policies and procedures, and specific risk assessment and control strategies. Interventions may include education and training in specific communication and behaviour management techniques targeting the diffusion and de-escalation of aggression, violence avoidance and breakaway strategies, and the physical restraint of aggressive people.

We will include interventions that are mandatory and voluntary; delivered at one-time or over multiple sessions; and delivered faceto-face, online or in blended form, including with synchronous or asynchronous components. We will include interventions delivered in workplace, educational and other professional settings. We will include stand-alone programmes and also those offered in conjunction with other organisational interventions, but only when those interventions are 'controlled for' in the analysis of impact or outcomes or can be determined not to have confounded or biased the results of the education and training intervention study.

\section{Types of outcome measures}

\section{Primary outcome}

- Number of episodes of aggression resulting in no harm or injury, psychological harm or injury, or physical harm or injury.

\section{Secondary outcomes}

- Personal knowledge, attitudes and skills relating to workplace aggression, including in relation to its prevention and minimisation. 
- Adverse personal and organisational outcomes attributable to incidents of workplace aggression (e.g. leave days taken, alterations to workforce participation including changing work patterns or attrition, litigation and rehabilitation costs).

\section{Search methods for identification of studies}

We will conduct a systematic literature search to identify all published and unpublished RCTs and CBAs that can be considered eligible for inclusion in this review. The literature search will identify potential studies in all languages. We will translate non-English language papers and fully assess them for potential inclusion in the review as necessary.

\section{Electronic searches}

We will search the following electronic databases from inception to date of search to identify potential studies:

- the Cochrane Central Register of Controlled Trials (CENTRAL) (The Cochrane Library);

- MEDLINE (PubMed);

- EMBASE;

- CINAHL (EBSCO);

- PsycINFO (ProQuest);

- NIOSHTIC (OSH-UPDATE);

- NIOSHTIC-2 (OSH-UPDATE);

- HSELINE (OSH-UPDATE);

- ISDOC (OSH-UPDATE).

We will use the keywords selected from the search strategy supplied in Appendix 1.

\section{Searching other resources}

We will also conduct a search of the following:

- ClinicalTrials.gov (www.ClinicalTrials.gov) and the World Health Organization (WHO) trials portal (www.who.int/ictrp/ en/);

- WorkSafe Australia;

- Canadian Centre for Occupational Health and Safety (CCOHS);

- The Campbell Collaboration and social, psychological, educational and criminological trials register.

Finally, we will check reference lists of all primary studies and review articles for additional references. We will contact experts in the field to identify additional unpublished materials.

\section{Data collection and analysis}

\section{Selection of studies}

Three review authors (JP, AH, SR) will independently screen titles and abstracts for inclusion of all the potential studies that we identify as a result of the search and code them as 'retrieve' (eligible or potentially eligible/unclear) or 'do not retrieve'. We will retrieve the full-text study reports/abstracts/publications and four review authors (HR, TD, SG, BM-J) will independently screen the full text and identify studies for inclusion, and, where a study is identified as being ineligible, we will record the reason/s. We will resolve disagreements by consensus or by involving another person from the review team (DH). We will identify and exclude duplicates and collate multiple reports of the same study so that each study, rather than each report, is the unit of interest in the review. We will record this selection process in sufficient detail to complete a PRISMA flow diagram and 'Characteristics of excluded studies' table.

\section{Data extraction and management}

We will use a study-specific data collection form for the collection of study characteristics, intervention details and outcome data (see Appendix 2). All review authors will pilot this on at least one study in the review. Three review authors (HR, AH, SG) will extract study characteristics from the identified included studies.

Using the study-specific data collection form, we will extract the following study characteristics.

- Publication details: authors, email address of corresponding author, date of publication, title, journal name, volume, issue and pages.

- Methods: study design (e.g. RCT/cluster RCT/CBA), including sampling, group allocation and treatment of missing data, study location/s, study setting/s and withdrawals.

- Participants: health worker type/s, total number of participants, number of health worker type sub-populations and proportions (\%), mean age or age range, gender, workplace/s (e.g. mental health, emergency department), work setting/s (e.g. hospital inpatient, hospital outpatient, community), work sector/s (e.g. public, private, non-government), and inclusion and exclusion criteria.

- Interventions: description of intervention and cointerventions, targeted knowledge, attitudes and skills, comparison, content of both intervention and control condition, and co-interventions (especially noting if bundled with other organisational interventions), duration, intensity, number commencing, number completing, adherence to protocol.

- Outcomes: description of primary and secondary outcomes specified and collected, measurement instruments used and validation status (e.g. reported/not reported), at which time points reported, controlling for biasing or confounding effects of co-interventions.

- Length of follow-up: time points at which primary and secondary outcomes were collected and categorisation to shortterm, medium-term and long-term follow-up (see further details 
below in Assessment of heterogeneity).

- Notes: funding for study, and notable conflicts of interest of trial authors.

Upon the conclusion of the final included list of studies, two review authors (DH, SR) will independently extract data from included studies. We will note in the 'Characteristics of included studies' table if outcome data were not reported in a usable way. One review author (TD) will transfer data into the Review Manager 5 (RevMan 2014). We will double-check that data are entered correctly by comparing the data presented in the systematic review with the study reports. A second review author (JP) will spot-check study characteristics for accuracy against the study report.

\section{Assessment of risk of bias in included studies}

Three review authors (DH, TD, BM-J) will independently assess risk of bias for each study using the criteria outlined in the Cochrane Handbook for Systematic Reviews of Interventions (Higgins 2011). We will resolve any disagreements by discussion or by involving another review author (AH, HR, JP, SG or SR). We will assess the risk of bias in the included RCTs according to the following domains.

- Random sequence generation.

- Allocation concealment.

- Blinding of participants and personnel.

- Blinding of outcome assessment.

- Incomplete outcome data.

- Selective outcome reporting.

- Other bias.

We will grade each potential source of bias as high, low or unclear and provide a quote from the study report together with a justification for our judgement in the 'Risk of bias' table. We will summarise the risk of bias judgements across different studies for each of the domains listed. We will consider blinding separately for different key outcomes where necessary (e.g. for unblinded outcome assessment, risk of bias for all-cause mortality may be very different than for a participant-reported pain scale). Where information on risk of bias relates to unpublished data or correspondence with a trialist, we will note this in the 'Risk of bias' table.

For CBAs, we will use a combination of the applicable domains of the risk of bias determination for RCTs and elements of the Downs and Black checklist (Downs 1998), as described in Chapter 13 of the Cochrane Handbook for Systematic Reviews of Interventions (Higgins 2011).

\section{Assessment of bias in conducting the systematic review}

We will conduct the review according to this published protocol and report any deviations from it in the 'Differences between protocol and review' section of the systematic review.

\section{Measures of treatment effect}

We will enter the outcome data for each study into the data tables in Review Manager 5 to calculate the treatment effects (RevMan 2014). We will use odds ratio/risk ratio/risk difference for dichotomous outcomes, and mean differences or standardised mean differences for continuous outcomes, or other type of data as reported by the authors of the studies. If only effect estimates and their 95\% confidence intervals or standard errors are reported in studies, we will enter these data into Review Manager 5 using the generic inverse-variance method. We will ensure that higher scores for continuous outcomes have the same meaning for the particular outcome, explain the direction to the reader and report where the directions were reversed if this was necessary. When the results cannot be entered in either way, we will describe them in the 'Characteristics of included studies' table, or enter the data into 'Additional tables'.

\section{Unit of analysis issues}

For studies that employ a cluster-randomised design and that report sufficient data to be included in the meta-analysis but do not make an allowance for the design effect, we will calculate the design effect based on a fairly large assumed intra-cluster correlation of 0.10 . We base this assumption of 0.10 being a realistic estimate by analogy on studies about implementation research (Campbell 2001). We will follow the methods stated in the Cochrane Handbook for Systematic Reviews of Interventions for the calculations (Higgins 2011).

\section{Dealing with missing data}

We will contact investigators or study sponsors in order to verify key study characteristics and obtain missing numerical outcome data where possible (e.g. when a study is identified as abstract only). Where this is not possible, and the missing data are thought to introduce serious bias, we will explore the impact of including such studies in the overall assessment of results by a sensitivity analysis.

If numerical outcome data are missing, such as standard deviations or correlation coefficients and we cannot obtain them from the authors, we will calculate them from other available statistics such as $\mathrm{P}$ values according to the methods described in the Cochrane Handbook for Systematic Reviews of Interventions (Higgins 2011).

\section{Assessment of heterogeneity}

We will assess the homogeneity of the results of all included studies based on similarity of intervention type and follow-up. We will consider interventions as different when they include education only or education combined with training.

We will categorise follow-up times of less than six months as shortterm follow-up, six months to 12 months as medium-term follow- 
up and greater than 12 months as long-term follow-up, and regard these as being different.

We will test for statistical heterogeneity using the $\mathrm{I}^{2}$ statistic ( Higgins 2011), using the following as a rough guide for interpretation: $0 \%$ to $40 \%$ might not be important; $30 \%$ to $60 \%$ may represent moderate heterogeneity; $50 \%$ to $90 \%$ may represent substantial heterogeneity and $75 \%$ to $100 \%$ considerable heterogeneity. In cases of substantial heterogeneity (defined as $\mathrm{I}^{2} \geq 50 \%$ ), we will explore the data further, including subgroup analyses, in an attempt to explain the heterogeneity.

\section{Assessment of reporting biases}

If we are able to pool 10 or more trials in any single meta-analysis, we will create and examine a funnel plot to explore possible smallstudy biases.

\section{Data synthesis}

Using Review Manager 5 software (RevMan 2014), we will pool data and display the results in separate forest plots by primary study design (i.e. we will analyse and display RCTs and CBAs separately) and we will pool data from studies judged to be homogeneous. If more than one study provides usable data in any single comparison, we will perform a meta-analysis. When studies are statistically heterogeneous, we will use a random-effects model. Otherwise, we will use a fixed-effect model. When using the random-effects model, we will conduct a sensitivity check by using the fixedeffect model to reveal differences in results. We will include a $95 \%$ confidence interval for all estimates.

We will describe skewed data reported as medians and interquartile ranges narratively.

Where multiple trial arms are reported in a single trial, we will include only the relevant arms. If two comparisons are combined in the same meta-analysis, we will halve the control group to avoid double-counting.

\section{'Summary of findings' table}

We will create a 'Summary of findings' table for each of the following outcomes:

- episodes of aggression resulting in no harm or injury, psychological harm or injury, or physical harm or injury.

- change in personnel knowledge, attitudes and skills relating to workplace aggression, including in relation to its prevention and minimisation.

- adverse personnel and organisational outcomes attributable to incidents of workplace aggression.

We will use the five GRADE considerations (study limitations, consistency of effect, imprecision, indirectness and publication bias) to assess the quality of a body of evidence as it relates to the studies that contribute data to the meta-analyses for the pre-specified outcomes. We will use methods and recommendations described in Section 8.5 and Chapter 12 of the Cochrane Handbook for Systematic Reviews of Interventions using GRADEpro software (GRADEPro 2014; Higgins 2011). We will justify all decisions to downgrade the quality of RCTs or upgrade the quality of CBAs using footnotes and we will make comments to aid reader's understanding of the review where necessary.

\section{Subgroup analysis and investigation of heterogeneity}

If we find a sufficient number of studies, we plan to carry out one or more of the following subgroup analyses where we identify substantial heterogeneity $\left(\mathrm{I}^{2}>50 \%\right)$ among included studies:

- face-to-face, online or blended delivery;

- length of intervention (delivered one-time or over multiple sessions).

\section{Sensitivity analysis}

If we find sufficient studies, we will a conduct a sensitivity analysis to test the robustness of our meta-analysis results by omitting studies that we judge to have a high risk of bias.

When using the random-effects model to combine data, we will conduct a sensitivity analysis by using the fixed-effect model to reveal differences in results.

Where we cannot obtain missing numerical outcome data and we think the missing data may introduce serious bias, we will explore the impact of including such studies in the overall assessment of results by a sensitivity analysis.

\section{Reaching conclusions}

We will base our conclusions only on findings from the quantitative or narrative synthesis of included studies for this review. We will avoid making recommendations for practice based on more than just the evidence, such as values and available resources. Our implications for research will suggest priorities for future research and outline what the remaining uncertainties are in the area.

\section{ACKNOW LEDGEMENTS}

We wish to acknowledge the contribution of Dr Maya Guest, formerly of the University of Newcastle, Australia (now retired), who originally conceived the protocol and led its early development, including in relation to the search strategy design in conjunction with the University of Newcastle librarians, and the contributions of Associate Professor Ashley Kable and Professor Michael Hazelton from the University of Newcastle, Australia, to the early development of the protocol. 
We thank Jani Ruotsalainen, Managing Editor, Cochrane Work Review Group for providing administrative and logistical support for the conduct of the current review, and Kaisa Neuvonen, Trials Search Co-ordinator, Cochrane Work Review Group for developing and testing the search strategies.

We would also like to thank the Cochrane Work Review Group's Co-ordinating Editor Jos Verbeek, Managing Editor Jani Ruotsalainen, Editors Thais Morata and Anneli Ojajärvi and external peer referees Joy Duxbury and Sabine Hahn for their comments. Last but not least, we thank Anne Lawson for copy editing the text.

\section{R E F E R E N C E S}

\section{Additional references}

\section{Anderson 2010}

Anderson L, FitzGerald M, Luck L. An integrative literature review of interventions to reduce violence against emergency department nurses. Journal of Clinical Nursing. Blackwell Publishing Ltd, 2010; Vol. 19, issue 17-18:2520-30.

\section{Archer 1988}

Archer J. The Behavioural Biology of Aggression. Cambridge: Cambridge University Press, 1988.

\section{Arimatsu 2008}

Arimatsu M, Wada K, Yoshikawa T, Oda S, Taniguchi H, Aizawa $Y$, et al. An epidemiological study of work-related violence experienced by physicians who graduated from a medical school in Japan. Journal of Occupational Health 2008; Vol. 50, issue 4:357-61.

\section{Arnetz 2001}

Arnetz JE, Arnetz BB. Violence toward health care staff and possible effects on the quality of patient care. Social Science \& Medicine 2001; Vol. 52, issue 3:417-27.

\section{Atawneh 2003}

Atawneh F, Zahid M, Al-Sahlawi K, Shahid A, Al-Farrah M. Violence against nurses in hospitals: prevalence and effects. British Journal of Nursing 2003; Vol. 12, issue 2:102-7.

\section{Beech 2006}

Beech B, Leather P. Workplace violence in the health care sector: a review of staff training and integration of training evaluation models. Aggression and Violent Behavior: a Review Journal 2006;11(1):27-43. [DOI: 10.1016/ j.avb.2005.05.004]

\section{Bowers 2006}

Bowers L, Nijman H, Allan T, Simpson A, Warren J, Turner LR. Prevention and management of aggression training and violent incidents on UK acute psychiatric wards. Psychiatric Services 2006; Vol. 57, issue 7:1022-6.

Briggs 2003

Briggs F, Broadhurst D, Hawkins R. Violence, threats and intimidation in the lives of professionals whose work involves child protection. A report on a research project funded by the Australian Criminology Research Council. CRC 15/01-02, 2003. crg.aic.gov.au/reports/20010215.pdf. Canberra: Australian Criminology Research Council, (accessed 2 August 2015).

\section{Brown 2011}

Brown LP, Rospenda KM, Sokas RK, Conroy L, Freels $\mathrm{S}$, Swanson NG. Evaluating the association of workplace psychosocial stressors with occupational injury, illness, and assault. Journal of Occupational and Environmental Hygiene. Taylor \& Francis, 2011; Vol. 8, issue 1:31-7.

\section{Bulatao 1996}

Bulatao EQ, VandenBos GR. Workplace violence: its scope and issues. In: VandenBos GR, Bulatao EQ editor (s). Violence on the Job: Identifying Risks and Developing Solutions. Washington, DC: American Psychological Association, 1996:1-23.

\section{Bushman 2001}

Bushman BJ, Anderson CA. Is it time to pull the plug on the hostile versus instrumental aggression dichotomy? Psychological Review 2001; Vol. 108, issue 1:273-9.

Camerino 2008

Camerino D, Estryn-Behar M, Conway PM, van Der Heijden BIJM, Hasselhorn H-M. Work-related factors and violence among nursing staff in the European NEXT study: a longitudinal cohort study. International Journal of Nursing Studies 2008; Vol. 45, issue 1:35-50.

\section{Campbell 2001}

Campbell MK, Mollison J, Grimshaw JM. Cluster trials in implementation research: estimation of intracluster correlation coefficients and sample size. Statistics in Medicine 2001;20(3):391-9.

\section{Campbell 2011}

Campbell JC, Messing JT, Kub J, Agnew J, Fitzgerald S, Fowler B, et al. Workplace violence: Prevalence and risk factors in the safe at work study. Journal of Occupational \& Environmental Medicine 2011; Vol. 53, issue 1:82-9.

\section{Carluccio 2010}

Carluccio A, Knychala V, Marshall C. Violence against frontline NHS staff: Research Study Conducted for COI on 
behalf of the NHS Security Management Service. London: NHS Security Management Service; 2010.

\section{Ceramidas 2010}

Ceramidas DM, Parker R. A response to patient-initiated aggression in general practice: Australian professional medical organisations face a challenge. Australian Journal of Primary Health 2010; Vol. 16, issue 3:252-9.

\section{Chappell 2006}

Chappell D, di Martino V. Violence at Work. 3rd Edition. Geneva: International Labour Office, 2006.

\section{Cherek 2003}

Cherek DR, Lane SD, Pietras CJ. Laboratory measures: point subtraction aggression paradigm. In: Coccaro EF editor(s). Aggression: Psychiatric Assessment and Treatment. New York: Marcel Dekker, Inc, 2003:215-48.

\section{Child 2010}

Child RJ, Mentes JC. Violence against women: the phenomenon of violence against nurses. Issues in Mental Health Nursing 2010;31(2):89-95. [DOI: 10.3109/ 01612840903267638]

\section{Cookson 2012}

Cookson H, Buckley P. Violence at Work: Findings from the 2010/11 British Crime Survey. London: Health Service Executive, 2012.

di Martino 2002

di Martino V. Workplace violence in the health sector. Country case studies Brazil, Bulgaria, Lebanon, Portugal, South Africa, Thailand and an additional Australian study. Synthesis report. Geneva: International Labour Office, International Council of Nurses, World Health Organization and Public Services International. Joint Programme on Workplace Violence in the Health Sector; 2002.

di Martino 2005

di Martino V. A cross-national comparison of workplace violence and response strategies. In: Bowie V, Fisher BS, Cooper CL editor(s). Workplace Violence: Issues, Trends, Strategies. Cullompton, UK: Willan Publishing, 2005: $15-36$.

Downs 1998

Downs SH, Black N. The feasibility of creating a checklist for the assessment of the methodological quality both of randomised and non-randomised studies of health care interventions. Journal of Epidemiology and Community Health 1998;52(6):377-84.

Dupré 2014

Dupré KE, Dawe KA, Barling J. Harm to those who serve: effects of direct and vicarious customer-initiated workplace aggression. Journal of Interpersonal Violence 2014;29(13): 2355-77. [DOI: 10.1177/0886260513518841]

\section{Estrada 2010}

Estrada F, Nilsson A, Jerre K, Wikman S. Violence at work the emergence of a social problem. Journal of Scandinavian Studies in Criminology and Crime Prevention. Routledge, 2010; Vol. 11, issue 1:46-65.

\section{Farrell 2005}

Farrell G, Cubit K. Nurses under threat: a comparison of content of 28 aggression management programs. International Journal of Mental Health Nursing 2005; Vol. 14:44-53.

Farrell 2006

Farrell GA, Bobrowski C, Bobrowski P. Scoping workplace aggression in nursing: findings from an Australian study. Journal of Advanced Nursing 2006; Vol. 55, issue 6: 778-87.

\section{Farrell 2010}

Farrell GA, Shafiei T, Salmon P. Facing up to 'challenging behaviour': a model for training in staff-client interaction. Journal of Advanced Nursing 2010; Vol. 66, issue 7: 1644-55.

\section{Flannery 2001}

Flannery RB. The employee victim of violence: recognizing the impact of untreated psychological trauma. American Journal of Alzheimer's Disease and Other Dementias 2001; Vol. 16, issue 4:230-3.

\section{Frank 1998}

Frank E, Brogan D, Schiffman M. Prevalence and correlates of harassment among US women physicians. Archives of Internal Medicine 1998; Vol. 158, issue 4:352-8.

Gascón 2009

Gascón S, Martínez-Jarreta B, González-Andrade JF, Santed Mlod Y, Rueda MA. Aggression towards health care workers in Spain: a multi-facility study to evaluate the distribution of growing violence among professionals, health facilities and departments. International Journal of Occupational and Environmental Health 2009;15(1):29-35.

Gates 2011

Gates DM, Gillespie G, Smith C, Rode J, Kowalenko T, Smith B. Using action research to plan a violence prevention program for emergency departments. Journal of Emergency Nursing 2011; Vol. 37, issue 1:32-9.

\section{Gerberich 2004}

Gerberich SG, Church TR, McGovern PM, Hansen HD, Nachreiner NM, Geisser MS, et al. An epidemiological study of the magnitude and consequences of work related violence: the Minnesota Nurses' Study. Occupational and Environmental Medicine 2004; Vol. 61, issue 6:495-503.

\section{Gerdtz 2013}

Gerdtz MF, Daniel C, Dearie V, Prematunga R, Bamert $\mathrm{M}$, Duxbury J. The outcome of a rapid training program on nurses' attitudes regarding the prevention of aggression in emergency departments: a multi-site evaluation. International Journal of Nursing Studies 2013;50(11): 1434-45. [DOI: 10.1016/j.ijnurstu.2013.01.007]

\section{GRADEPro 2014 [Computer program]} McMaster University. GRADEpro [Computer program on www.gradepro.org]. McMaster University, 2014.

\section{Guay 2014}

Guay S, Goncalves J, Jarvis J. Verbal violence in the workplace according to victims' sex - a systematic review of 
the literature. Aggression and Violent Behaviour 2014;19(5): 572-78. [DOI: 10.1016/j.avb.2014.08.001]

\section{Hahn 2008}

Hahn S, Zeller A, Needham I, Kok G, Dassen T, Halfens RJG. Patient and visitor violence in general hospitals: a systematic review of the literature. Aggression and Violent Behavior 2008; Vol. 13, issue 6:431-41.

\section{Hahn 2010}

Hahn S, Müller M, Needham I, Dassen T, Kok G, Halfens RJ. Factors associated with patient and visitor violence experienced by nurses in general hospitals in Switzerland: a cross-sectional survey. Journal of Clinical Nursing 2010;19(23-24):3535-46. [DOI: 10.1111/ j.1365-2702.2010.03361.x]

Hahn 2013

Hahn S, Müller M, Hantikainen V, Kok G, Dassen T, Halfens RJ. Risk factors associated with patient and visitor violence in general hospitals: results of a multiple regression analysis. Journal of International Nursing Studies 2013;50 (3):374-85. [DOI: 10.1016/j.ijnurstu.2012.09.018]

\section{Harrell 2011}

Harrell E. Workplace violence, 1993-2009: National crime victimization survey and the census of fatal occupational injuries. NCJ 233231. Washington: Bureau of Justice Statistics; 2011.

Heckemann 2015

Heckemann B, Zeller A, Hahn S, Dassen T, Schols JM, Halfens RJ. The effect of aggression management training programmes for nursing staff and students working in an acute hospital setting. A narrative review of current literature. Nurse Education Today 2015;35(1):212-19. [DOI: 10.1016/j.nedt.2014.08.003]

\section{Hegney 2006}

Hegney D, Eley R, Plank A, Buikstra E, Parker V. Workplace violence in Queensland, Australia: the results of a comparative study. International Journal of Nursing Practice 2006; Vol. 12, issue 4:220-31.

\section{Heponiemi 2014}

Heponiemi T, Kouvonen A, Virtanen M, Vänskä J, Elovainio M. The prospective effects of workplace violence on physicians' job satisfaction and turnover intentions: the buffering effect of job control. BMC Health Services Research 2014;14(19):1-8. [DOI: 10.1186/1472-6963-14-19]

\section{HEPRU 2003}

Health Policy and Economic Research Unit, British Medical Association. Violence at work: the experience of UK doctors. London: British Medical Association; 2003.

\section{HEPRU 2008}

Health Policy and Economic Research Unit, British Medical Association. Violence in the workplace: The experience of doctors in Great Britain. London: British Medical Association; 2008

\section{Hershcovis 2010}

Hershcovis MS, Barling J. Toward a multi-focal approach to workplace aggression: a meta-analytic review of outcomes from different perpetrators. Journal of Organizational Behavior 2010; Vol. 31, issue 1:24-44.

\section{Higgins 2011}

Higgins JPT, Green S (editors). Cochrane Handbook for Systematic Reviews of Interventions Version 5.1.0 [updated March 2011]. The Cochrane Collaboration, 2011. Available from www.cochrane-handbook.org.

\section{Hills 2008}

Hills D. Relationships between aggression management training, perceived self-efficacy and rural general hospital nurses' experiences of patient aggression. Contemporary Nurse 2008; Vol. 31, issue 1:20-31.

\section{Hills 2012}

Hills D, Joyce C, Humphreys J. A national study of workplace aggression in Australian clinical medical practice. Medical Journal of Australia 2012; Vol. 197, issue 6: 336-40.

\section{Hills 2013}

Hills D, Joyce C. A review of research on the prevalence, antecedents, consequences and prevention of workplace aggression in clinical medical practice. Aggression and Violent Behavior: a Review Journal 2013; Vol. 18, issue 5: 554-69.

\section{Hills 2014}

Hills D, Joyce C. Workplace aggression in clinical medical practice: associations with job satisfaction, life satisfaction and self-rated health. Medical Journal of Australia 2014;201 (9):535-40.

\section{Hinduja 2007}

Hinduja S. Workplace violence and negative affective responses: a test of Agnew's general strain theory. Journal of Criminal Justice 2007; Vol. 35, issue 6:657-66. [0047-2352]

\section{Hodgson 2004}

Hodgson MJ, Reed R, Craig T, Murphy F, Lehmann L, Belton L, et al. Violence in healthcare facilities: lessons from the Veterans Health Administration. Journal of Occupational \& Environmental Medicine 2004; Vol. 46, issue 11:1158-65.

\section{Hogh 2005a}

Hogh A, Henriksson M, Burr H. A 5-year follow-up study of aggression at work and psychological health. International Journal of Behavioral Medicine. Springer Boston, 2005; Vol. 12, issue 4:256-65. [1070-5503]

\section{Hogh 2005b}

Hogh A, Viitasara E. A systematic review of longitudinal studies of nonfatal workplace violence. European Journal of Work \& Organizational Psychology. Psychology Press (UK), 2005; Vol. 14, issue 3:291-313. [1359432X]

\section{ILO 2002}

Joint Programme on Workplace Violence in the Health Sector. Framework guidelines for addressing workplace violence in the health sector. Geneva: International Labour Office; 2002. 


\section{ILO 2003}

International Labour Organization Sectoral Activities Programme. Code of practice on workplace violence in services sectors and measures to combat this phenomenon. Geneva: International Labour Organization; 2003.

Judy 2009

Judy K, Veselik J. Workplace violence: a survey of paediatric residents. Occupational Medicine 2009; Vol. 59, issue 7: 472-5.

Kable 2012

Kable A, Guest M, McLeod M. Resistance to care: contributing factors and associated behaviours in healthcare facilities. Journal of Advanced Nursing 2012; Vol. 69, issue $8: 1747-60$

\section{Kansagra 2008}

Kansagra SM, Rao SR, Sullivan AF, Gordon JA, Magid DJ, Kaushal R, et al. A survey of workplace violence across 65 U.S. emergency departments. Academic Emergency Medicine. Blackwell Publishing Ltd, 2008; Vol. 15, issue 12:1268-74. [1553-2712]

\section{Kvas 2014}

Kvas A, Seljak J. Unreported workplace violence in nursing. International Nursing Review 2014;61(3):344-51. [DOI: 10.1111/inr.12106]

\section{Laker 2010}

Laker C, Gray R, Flach C. Case study evaluating the impact of de-escalation and physical intervention training. Journal of Psychiatric and Mental Health Nursing 2010; Vol. 17, issue 3:222-8.

\section{Lanctôt 2014}

Lanctôt N, Guay S. The aftermath of workplace violence among healthcare workers: a systematic literature review of the consequences. Aggression and Violent Behavior 2014;19 (5):492-501. [DOI: 10.1016/j.avb.2014.07.010]

Lapierre 2005

Lapierre LM, Spector PE, Leck JD. Sexual versus nonsexual workplace aggression and victims' overall job satisfaction: a meta-analysis. Journal of Occupational Health Psychology 2005;10(2):155-69.

\section{Laschinger 2014}

Laschinger HK. Impact of workplace mistreatment on patient safety risk and nurse-assessed patient outcomes. Journal of Nursing Administration 2014;44(5):284-90. [DOI: $10.1097 /$ NNA.0000000000000068]

\section{LeBlanc 2002}

LeBlanc MM, Kelloway EK. Predictors and outcomes of workplace violence and aggression. Journal of Applied Psychology 2002; Vol. 87, issue 3:444-53.

\section{LeBlanc 2006}

LeBlanc MM, Dupre KE, Barling J. Public-initiated violence. In: Kelloway EK, Barling J, Hurrell Jr. JJ editor (s). Handbook of Workplace Violence. Thousand Oaks: Sage Publications, Inc, 2006:261-80.

\section{Lewis 2005}

Lewis SE, Orford J. Women's experiences of workplace bullying: Changes in social relationships. Journal of
Community \& Applied Social Psychology. John Wiley \& Sons, Ltd., 2005; Vol. 15, issue 1:29-47.

\section{Livingston 2010}

Livingston JD, Verdun-Jones S, Brink J, Lussier P, Nicholls T. A narrative review of the effectiveness of aggression management training programs for psychiatric hospital staff. Journal of Forensic Nursing 2010; Vol. 6, issue 1:15-28.

\section{Martínez-Jarreta 2007}

Martínez-Jarreta B, Gascón S, Santed MA, Goicoechea J. Medical-legal analysis of aggression towards health professionals. An approach to a silent reality and its consequences on health [Análisis médico-legal de las agresiones a profesionales sanitarios. Aproximación a una realidad silenciosa y a sus consecuencias para la salud]. Medicina Clínica 2007;128(8):307-10.

\section{Mayhew 2000}

Mayhew C. Preventing client initiated violence: a practical handbook. Canberra: Australian Institute of Criminology; 2000.

\section{Mayhew 2001}

Mayhew C, Chappell D. Prevention of occupational violence in the health workforce. Working paper series 140 Taskforce on the Prevention and Management of Violence in the Health Workplace, Discussion Paper No. 2. Sydney: School of Industrial Relations and Organizational Behaviour and Industrial Relations Research Centre, University of New South Wales; 2001.

\section{Mayhew 2004}

Mayhew C. Occupational violence/bullying in the health industry. In: McCarthy P, Mayhew C editor(s). Safeguarding the Organization Against Violence and Bullying: an International Perspective. Houndmills, UK: Oalgrave Macmillan, 2004:110-28.

\section{Mayhew 2007}

Mayhew C, Chappell D. Workplace violence: an overview of patterns of risk and the emotional/stress consequences on targets. International Journal of Law and Psychiatry 2007; Vol. 30, issue 4-5:327-39.

\section{McCarthy 2004}

McCarthy P. The safeguarding challenge. In: McCarthy P, Mayhew C editor(s). Safeguarding the Organization Against Violence and Bullying: an International Perspective. Houndmills, UK: Oalgrave Macmillan, 2004:1-16.

\section{Nachreiner 2005}

Nachreiner NM, Gerberich SG, McGovern PM, Church TR, Hansen HE, Geisser MS, et al. Impact of training on work-related assault. Research in Nursing \& Health 2005; Vol. 28, issue 1:67-78.

\section{Needham 2005}

Needham I, Abderhalden C, Halfens RJ, Dassen T, Haug HJ, Fischer JE. The effect of a training course in aggression management on mental health nurses' perceptions of aggression: a cluster randomised controlled trial. International Journal of Nursing Studies 2005; Vol. 42, issue 6:649-55. 


\section{NICE 2006}

National Institute for Health and Clinical Excellence.

Violence clinical practice guidelines: the short-term management of disturbed/violent behaviour in in-patient psychiatric settings and emergency departments. London: Royal College of Nursing; 2006.

Niedhammer 2009

Niedhammer I, David S, Degioanni S, Drummond A, Philip P. Workplace bullying and sleep disturbances: findings from a large scale cross-sectional survey in the French working population. Sleep 2009; Vol. 32, issue 9: 1211-9.

\section{O'Brien-Pallas 2009}

O'Brien-Pallas L, Wang S, Hayes L, Laporte D. Creating work environments that are violence free. World Hospitals and Health Services 2009; Vol. 45, issue 2:12-8.

\section{OSHA 2004}

Occupational Safety and Health Administration, US Department of Labor. Guidelines for preventing workplace violence for health care \& social service workers. Washington: US Department of Labor; 2004.

\section{Packham 2011}

Packham C. Violence at work: Findings from the 2009/10 British crime survey. London: Health Service Executive; 2011.

\section{Paice 2009}

Paice E, Smith D. Bullying of trainee doctors is a patient safety issue. The Clinical Teacher 2009;6(1):13-17. [DOI: 10.1111/j.1743-498X.2008.00251.x]

\section{Parent-Thirion 2007}

Parent-Thirion A, Marcias EF, Hurley J, Vermeylen G. Fourth European working conditions survey. Dublin: European Foundation for the Improvement of Living and Working Conditions; 2007.

\section{Parker 2010}

Parker RM, Ceramidas DM, Forrest LE, Herath PM, McRae I. Patient initiated aggression and violence in the Australian general practice setting. Canberra: The Australian Primary Health Care Institute; 2010.

\section{Price 2015}

Price O, Baker J, Bee P, Lovell K. Learning and performance outcomes of mental health staff training in de-escalation techniques for the management of violence and aggression. British Journal of Psychiatry 2015;206(6):447-55. [DOI: 10.1192/bjp.bp.114.144576]

\section{RevMan 2014 [Computer program]}

The Nordic Cochrane Centre, The Cochrane Collaboration. Review Manager (RevMan). Version 5.3. Copenhagen: The Nordic Cochrane Centre, The Cochrane Collaboration, 2014.

\section{Richter 2006}

Richter D, Needham I, Kunz S. The effects of aggression management training for mental health care and disability care staff: a systematic review. In: Richter D, Whittington R editor(s). Violence in Mental Health Settings. New York: Spring Science and Business Media, LLC, 2006:211-27.

\section{Roche 2010}

Roche M, Diers D, Duffield C, Catling-Paull C. Violence toward nurses, the work environment, and patient outcomes. Journal of Nursing Scholarship 2010; Vol. 42, issue 1:13-22.

\section{Rosenstein 2008}

Rosenstein AH, O'Daniel M. A survey of the impact of disruptive behaviors and communication defects on patient safety. Joint Commission Journal on Quality and Patient Safety 2008;34(8):464-71.

\section{Spector 2014}

Spector PE, Zhou ZE, Che XX. Nurse exposure to physical and nonphysical violence, bullying, and sexual harassment: a quantitative review. International Journal of Nursing Studies 2014; Vol. 51:72-84.

\section{Taylor 2010}

Taylor JL, Rew L. A systematic review of the literature: workplace violence in the emergency department. Journal of Clinical Nursing 2010; Vol. 20:1072-85.

\section{Ventura-Madangeng 2009}

Ventura-Madangeng J, Wilson D. Workplace aggression experienced by registered nurses: a concept analysis. Nursing Praxis in New Zealand 2009;25(3):37-50.

\section{Viitasara 2002}

Viitasara E, Menckel E. Developing a framework for identifying individual and organizational risk factors for the prevention of violence in the health-care sector. Work. IOS Press, 2002; Vol. 19, issue 2:117-23.

\section{Viitasara 2003}

Viitasara E, Sverke M, Menckel E. Multiple risk factors for violence to seven occupational groups in the Swedish caring sector. Industrial Relations 2003; Vol. 58, issue 2:202-31.

\section{Wieclaw 2006}

Wieclaw J, Agerbo E, Mortensen PB, Burr H, Tüchsen F, Bonde JP. Work related violence and threats and the risk of depression and stress disorders. Journal of Epidemiology and Community Health 2006; Vol. 60, issue 9:771-5.

\section{Wiskow 2003}

Wiskow C. Guidelines on workplace violence in the health sector. Comparison of major known national guidelines and strategies: United Kingdom, Australia, Sweden, USA (OSHA and California). Geneva: World Health Organization; 20032003.

* Indicates the major publication for the study 
A P P E N D I CES

\section{Appendix I. MEDLINE search strategy}

Databases searched:

- PubMed

Symbols used in this document:

\begin{tabular}{ll}
\hline mp & Keyword search in Ovid databases. This is the broadest search possible \\
\hline ab & Abstract \\
\hline adj & Adjacency search in Ovid databases - "adj3" will retrieve the nominated words within 3 words of each other, in any order \\
\hline exp & Exploded search in Ovid Databases \\
\hline MeSH & Medical subject heading \\
\hline mh & Main heading \\
\hline ti & Title \\
\hline tw & An alias for all of the fields in a database that contain text words and that are appropriate for a subject search \\
\hline I & A slash appearing after a search word/phrase in OVID databases indicates search within the subject heading field \\
\hline$*$ & Truncation symbol - will retrieve all words beginning with the set of letters appearing before the symbol \\
\hline
\end{tabular}

Database: PubMed

Name of Host: Ovid

Number of results: 392 (RCT) plus 200 (CBA)

Date searched: 3 March 2015

Conducted by: Kaisa Neuvonen

\begin{tabular}{ll} 
Set \# & Search string \\
\hline 1 & "Workplace Violence"[Mesh] OR "Vi- \\
& olence/prevention and control"[Mesh] \\
& OR violence[tw] OR violent[tw] OR \\
& "Aggression"[Mesh] OR aggression*[tw] \\
& OR angry[tw] OR "Hostility"[Mesh] \\
& OR hostil*[tw] OR "inappropriate \\
behavior"[tw] OR "Agonistic Behav- & ior"[Mesh] OR "Bullying"[Mesh] OR \\
bully*[tw] OR mob*[tw] OR ha- \\
rass*[tw] OR pester*[tw] OR dis- \\
rupt*[tw] OR incivility[tw] OR "emo-
\end{tabular}

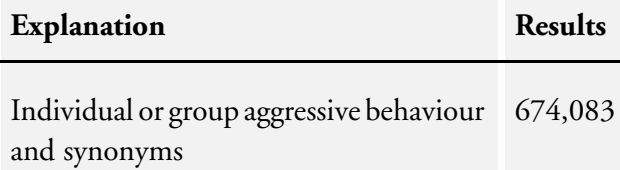


tional-verbal abuse"[tw] OR abus*[tw]

OR assault ${ }^{*}[\mathrm{tw}]$

2 work-related

OR at work[tw] OR "Work" [Mesh] OR

Place of employment and synonyms $\quad 1,360,864$

work[tw] OR worke*[tw] OR work-

place $^{*}[\mathrm{tw}]$ OR work place* $[\mathrm{tw}] \mathrm{OR}$

work site*[tw] OR occupation*[tw] OR

"Occupations" $[\mathrm{MeSH}]$ OR "Occupa-

tional Groups" $[\mathrm{MeSH}]$ OR job*[tw]

OR “Occupational Health” [MeSH] OR

"occupational health"

4 "Health Personnel”[Mesh] OR "Person-

The range of health workers that may be $1,081,347$ nel, Hospital"[Mesh] OR "health care the targets of workplace aggression worker" $[\mathrm{tw}] \mathrm{OR}$ "health care workers" [tw] OR "health care personnel" [tw] OR

"health personnel" [tw] OR "health-personnel" [tw] OR "health provider" [tw] OR "health providers" [tw] OR "health care provider" [tw] OR "health care providers" [tw] OR "health staff" [tw] OR "health care staff" [tw] OR "healthcare staff" [tw] OR "health professional" [tw] OR "health care professional" $[\mathrm{tw}]$ OR "healthcare professional" [tw] OR "health worker" [tw] OR "medical staff" [tw] OR "medical personnel" $[\mathrm{tw}]$ OR "medical professional" [tw] OR "medical worker" $[\mathrm{tw}]$ OR "medical workers" [tw] OR "medical provider" [tw] OR "military-medical personnel" [tw] OR "Physicians"[Mesh] OR "physician" $[\mathrm{tw}]$ OR "physicians" [tw] OR "doctor" [tw] OR "practitioner" [tw] OR "clinician" $[\mathrm{tw}] \mathrm{OR}$ "nursing staff” [tw] OR "Nurses"[Mesh] OR “nurse" [tw] OR "nurses" [tw] OR "nursing assistant" [tw] OR "nursing assistants" [tw] OR “Nurses' Aides”[Mesh] OR “Nurse Midwives”[Mesh] OR “midwife" [tw] OR "midwives" [tw] OR "dental personnel” [tw] OR “dental staff” [tw] OR “Dentists" [Mesh] OR “dentist”[tw] OR "dentists" [tw] OR "dental assistant” [tw] OR "dental assistants" [tw] OR 
"Dental Assistants"[Mesh] OR "Pharmacists"[Mesh] OR "pharmacist" [tw] OR "Physical Therapists"[Mesh] OR "physical therapist"[tw] OR "physical therapists" $[\mathrm{tw}]$ OR "physiotherapist" [tw] OR "physiotherapists" [tw] OR "therapist" [tw] OR "therapists" [tw] OR "Physical Therapist Assistants"[Mesh] OR

"technician" [tw] OR "technicians" [tw] OR “radiographer" [tw] OR "radiographers" [tw] OR “emergency medical services" [tw] OR "Emergency Medical Services"[MeSH] OR "transporting patients" [tw] OR "patient transport"[tw] OR "Ambulances" [Mesh] OR "Allied Health Personnel"[Mesh] OR "paramedic" [tw] OR "paramedics" [tw] OR “paramedical personnel” [tw] OR "health manager" $[\mathrm{tw}] \mathrm{OR}$ "health care manager" $[\mathrm{tw}]$ OR "healthcare manager" [tw] OR "clinical officer" [tw] OR "reception"[tw]

$5 \quad$ \#3 AND \#4

6 "Health Personnel/education" [Mesh] OR "Nursing Staff, Hospital/education" [Mesh] OR "Health Occupations/education" [Mesh] OR education[tw] OR "Inservice Training"[Mesh] OR training[tw] OR inservice[tw] OR in-service[tw] OR "Staff Development"[Mesh] OR program* OR "aggression management"
$7 \quad$ \#5 AND \#6
Workplace aggression directed toward 24,297

health workers

Workplace education and training pro- $1,571,227$

grammes and interventions
Education and training for workplace 9,608 aggression directed toward health work-

ers

8 ("Comparative

Studies evaluating education and train- $8,438,469$

Study” [Publication Type] OR effectiveness OR program OR intervention OR ing programmes reduction OR effect*[ti] OR evaluation OR decrease* OR "prevention and control” OR measures OR improve*[tiab]) 
$9 \quad \# 7$ AND \#8

10 (randomized controlled trial $[\mathrm{pt}] \mathrm{OR}$ controlled clinical trial [pt] OR randomized [tiab] OR placebo [tiab] OR clinical trials as topic [mesh: noexp] OR randomly [tiab] OR trial [ti] NOT (animals [mh] NOT humans [mh]))
Studies evaluating the education and 5,779

training programmes

RCTs

878,923
11 \#9 AND \#10

trolled Before-After Studies”[Mesh] OR

"controlled before-after study" [tw] OR

"controlled before-after studies" [tw] OR

"CBA study" OR "CBA studies" OR

"before-after study" [tw] OR "before-after studies" [tw] OR "Prospective Studies"[Mesh] OR prospective study OR

"longitudinal studies" [MeSH]
RCTs evaluating the education and 392

training programmes
CBAs $\quad 595,968$

CBAs evaluating the education and 200 training programmes

\section{Appendix 2. Data extraction form}

\begin{tabular}{ll}
\hline Categories & Sub-categories \\
\hline Publication details & $\begin{array}{l}\text { Authors and email address of corresponding author } \\
\text { Date of publication } \\
\text { Title } \\
\text { Journal name, volume, issue and pages }\end{array}$ \\
\hline Methods & $\begin{array}{l}\text { Study design (e.g. RCT/cluster RCT/CBA) including sampling, group allocation and treatment of missing } \\
\text { data } \\
\text { Study location/s } \\
\text { Study setting/s } \\
\text { Withdrawals }\end{array}$ \\
\hline Participants & $\begin{array}{l}\text { Health worker type/s } \\
\text { Total number, number of health worker type sub-populations and proportions (\%) } \\
\text { Mean age or age range } \\
\text { Gender }\end{array}$ \\
\hline
\end{tabular}


Workplace/s (e.g. mental health, emergency department)

Work setting/s (e.g. hospital inpatient, hospital outpatient, community)

Work sector/s (e.g. public, private, non-government)

Inclusion and exclusion criteria

Intervention/s

Description of intervention and co-interventions (especially noting if bundled with other organisational interventions)

Targeted knowledge, attitudes and skills

Comparison

Content of both intervention and control condition, and co-interventions

Duration

Intensity (e.g. frequency or levels of intervention)

Number commencing

Number completing

Adherence to protocol

Outcomes

Description of primary and secondary outcomes specified and collected

Measurement instruments used and validation status (e.g. reported/not reported)

Time points reported

Controlling for biasing or confounding effects of co-interventions

Length of follow-up Time points at which primary and secondary outcomes were collected and categorisation to short-term $(<6$ months), medium-term (6-12 months) and long-term (> 12 months) follow-up

Notes

Funding for study

Notable conflicts of interest of study authors

\section{CONTRIBUTIONSOFAUTHORS}

Co-ordinating the protocol: $\mathrm{DH}$.

Designing the protocol: DH, HR, JP, AH, TD, SR.

Designing search strategies: DH, TD in collaboration with Kaisa Neuvonen, Trials Search Co-ordinator of the Cochrane Occupational Safety and Health Group.

Writing the protocol: DH, HR, JP, AH, TD, SR, SG, BM-J.

Providing general advice on the protocol: DH, HR, JP, AH, TD, SR, SG, BM-J. 


\section{DECLARATIONSOF INTEREST}

Danny Hills: Over the past three years, I have been employed by three Universities to undertake teaching and research work, largely unrelated to this Cochrane review. I have also received a PhD scholarship to undertake full-time and part-time research on workplace aggression in Australian clinical medical practice, and have had numerous reports on workplace aggression in nursing and medical practice published in Australian and international journals.

Heather Ross: I have received funding from my own institution to attend a Cochrane review training session.

Jacqueline Pich: None known.

April Hill: None known.

Therese Dalsbø: None known.

Sanaz Riahi: None known.

Stephane Guay: None known.

Begoña Martínez-Jarreta: None known. 